forty to ninety grains of the powdered collinsonia root will, in many cases, render any operative procedure unnecessary.

The first patient was a woman, aged 45 , who suffered for years from darting pains in the rectum, especially severe just before defæcation. She has visited numerous celebrated springs, and consulted several regular and irregular physicians without obtaining marked relief. When she placed herself under my care I instituted a general tonic, anti-neuralgic treatment, combined with suitable laxatives. $\mathrm{Her}$ general health improved almost immediately, but the local disorder remained stubborn. Finally, suspecting the existence of an anal fissure or ulcer, I made an examination of the rectum, but discovered nothing abnormal but the vice-like grasp in which the finger or the speculum was held by the sphincter muscle.

Suppositories composed of opium, opium and belladonna, opium and quinine were then ordered in succession, and successively failed to more than temporarily relieve. I then decided to either divulse or incise the sphincter; but, being desirous to ascertain the relative merits of the operations, I ordered her as a placebo four suppositories, each containing thirty grains of powered collinsonia, with instructions to use one every night as usual. To my surprise and gratification the patient appeared at my office two days afterwards, and in formed me that "the last cartridges which I had ordered for her had given her so much ease, that she used two a day instead of one, and wanted more of the same kind, as she was convinced that I had found the right .cure at last." I questioned her closely, and found that her relief was genuine and not assumed, and that it began within an hour after using the first suppository. I then ordered eight more, containing forty grains each instead of thirty, and directed her to use one night and morning. She returned on the sixth day, and told me that after having used five, she felt so well that she did not think it necessary to finish the rest. The improvement was complete and permanent. During the year and a half that has elapsel since then, she has consulted me twice for other troubles, but the rectal pain has never reappeared.

The second case was a woman, aged 35 , and was similar to the preceding one, and was cured in a week by collinsonia suppositories

The third case was that of a young man, aged 19, who suff 9 red from violent headache every second day. Inquiry revealed the fact that the headache was almost invariably accompanied by a feeling of weight in the abdomen, and a desire to defæcate, which could only be accomplished by much straining and repeated efforts, extending sometimes over a period of from twenty to forty minutes; at the expiration of that time a large evacuation of the bowels usually occurred, followed by the immediate disappearance of the sense of weight in the abdomen, and the gradual cessation of the headache. The patient had taken all the varieties of pills in the market without any appreciable benefit, except when they left his stools thin and watery.

As defecation was not preceded or accompanied by pain, and usually relieved the other symptoms, I concluded that there was no ulceration or fissure present, and that the trouble was due solely to spasmodic stricture of the sphincter ani. I ordered him four suppositories, containing one drachm each of powdered collinsonia, one to be used each night at bedtime. He returned in four days as bad as ever, and inclined to doubt the value of "those things." I encouraged him to try again, and ordered six more, each containing seventy-five grains. I did not see him again for two weeks, when he came back and stated that when he had used four he had felt so good that he threw the other two away, but that the trouble had returned, and was now worse than ever. I then ordered eight suppositories with ninety grains of collinsonia in each, and directed him to use one every second night, regardless of how well he might be. He obeyed these instructions faithfully, and during the five months which have since passed by he has not had any symptoms of his former troubles.

It is more than probable that vaginismus or spasms of the sphincter vaginx can be resdily and safely relieved without resorting to the ludicrous or painful methods narrated in the text-books, by thecontinued employment of raginal suppositories of collinsonia; opium, belladonna, chloral, conium, hyoscyamus, or stramonium may be added if advisable.

The anti-spasmodic properties of collinsonia render it of value in flatulent colic, infantile colic, and biliary colic. It is especially serviceable in the latter affection, if given in the form of warm infusions so as to thoroughly relax the biliary passayes and facilitate the onward movement of the irritative calculi.

The most available preparation in colic is the tincture, the dose of which ranges from ten drops to half a drachm for children, and from one to two drachms frequently repeated for adults. Collinsonia alono will be found quickly curative in many cases of colic when unaccompanied by rise of temperature. It will not relieve lead colic, or the pain of peritonitis or entero-colitis.
Collinsonia has proved curative in my hands in two cases of gastralgia, in which morphine, cannabis indica, belladonna, and various other remedies only gave temporary relief. I also obtained markedly beneficial results from its use in five cases of dysmenorrhœea. Each patient was directed to take half a drachm of the fluid extract three times a day for a week before the appearance of the menses, and two drachms of the tincture every four hours during their continuance. In the first and fifth cases the pain though previously severe was not noticeable, in the remaining cases it was so much lessened that the patients pursued their daily avocations as usual, one as a seamstress, one as a saleswoman, and the other as a telegraph operator, instead of being compelled to stay in bed for two or more days.

Ordinary colds and mild attacks of lumbago can bo quickly broken up by taking a cupful of a hot infusion of collinsonia at bedtime. Spasmodic croup can be immediately relieved by the same means.

Collinsonia is an effective remedy in relaxation of the uvula, chronic pharyngitis and hoarseness dependent upon a lack of tonicity of the vocal chords. The fluid extract may be given in these affections in half-drachm doses four times daily, and employed also as a gargle when dilnted with four times its volume of water. It is also of value in gastro-intestinal catarrh and the catarrhal gastritis of beer and alcohol drinkers. It lessens the desire for liquor, restrains the secretion of mucus, and restores the normal tone of the alimentary canal, and reinvigorates the depressed nervous system.

Collinsonia is equal, if not superior; to cinucifuga in the treatment of chorea, and may be substituted for arsenic with advantage in many cases of that disease occurring in infancy and early childhood. In three cases recently treated with collinsonia, all traces of the disease disappeared in from two to four weeks. It is but fair to state that the same remedy failed to make any impression on two previous cases.

Collinsonia will be found palliative, if not curative, in whoopingcough, and may safely be given in that affection without the dread of disordening the stomach, or producing any other unpleasant symptoms. It may also be given with confidence in nervous cough, and the irritative cough of pharyngeal catarrh.

Collinsonia is also of value in moderate doses as a mild but certain general tonic, increasing the appetite, promoting digestion, and gently stimulating all the organs of excretion. It may be given with decided benefit in anæmia, chlorosis, incipient phthisis, and convales. cence from the various eruptive fevers.

Externally, it constitutes an excellent application to contused and incised wounds. Indolent ulcers may be stimulated to healthy action by an ointment consisting of one drachm of powdered collinsonia and one ounce of fresh lard. $\Delta$ scarides may be effectually destroyed by rectal injection composed of the fluid extract diluted with four parts of water.

\section{A PRELIMINARY REPORT OF EXPERIMENTAL RESEARCHES CONCERNING THE INFECTIOUS NATURE OF TRAUMATIC TETANUS.}

Read in the Section of Pathology of the Ninth International Medical Congress, September 6th, 1887.

By EDWARD O. SHAKESPEARE, A.M., M.D., Pathologist to the Philadelphia Hospital, etc.

THE author reported in detail a long series of experiments which are still in progress, and announced the results already obtained. Upwards of fifty inoculations have already been made. Two methods of inoculation have been employed. Intra-cranial inoculations after the method of Pasteur in the case of rabies, and subcutaneous or intermuscular injections by means of hypodermic syringes. The inoeula. tions were always made with thorough antiseptic precautions and with sterilised instruments. In none of the experiments was there any sign of accidental infection, such as suppuration, etc. The material used for inoculation was in general obtained from the medulla or the spinal cord, and cultures in neutral or slightly alkaline flesh-glycerineagar recommended by Roux for the culture of tubercle bacilli. The tetanus material was taken, under aseptic precautions, from a horse and a mule dead of traumatic tetanus in the Veterinary Department of the University of Pennsylvania, the brain, medulla, and cord being removed one and three. hours respectively after death, and immediately kept on ice until used. The inoculation material was in general prepared in the following manner : A small piece of the medulla or cord was thoroughly rubbed up in sterilised distilled water; after, the solid particles were allowed for a few minutes to subside to the bottom of the vessel; the opalescent emulsion thus obtained was drawn off by 
means of sterilised pipettes, and placed in small sterilised vials until used, never having been thus kept longer than three hours before inoculation. Eight control experiments were made. The author concludes his paper as follows :

\section{SUMMARY OF RESULTS.}

First Series. - Eight rabbits were inoculated sub durd cerebri between August 1st and 18th inclusive, from a horse dead of tetanus. The rabbit inoculated direstly from this horse showed the first symptoms of tetanus within fifteen hours, and died of well-marked tetanus within forty-eight hours after inoculation. Both the period of incubation and that of death became markedly shortened in continuing the inoculations from rabbit to rabbit.

Second Series.-.Four rabbits were inoculated sub durâ cerebri from the same medulla of horse. The rabbit inoculated directly from the horse showed the first symptoms of tetanus within twenty hours, and died within forty-eight hours after. Continuing the inoculation from rabbit to rabbit, the period of incubation and of death became markedly shortened.

Third Series. - Four rabbits were inoculated sub durâ cerebri from the same medulla of horse after it had been kept on ice a day longer. The rabbit inoculated directly from the horse showed the first symptoms of tetanus within twenty-four hours, and died within fortyeight hours after inoculation. Continuing the inoculations from rabbit to rabbit, the period of incubation and of death became markedly shortened.

Fourth Series. - Three rabbits were inoculated sub durâ cerebri from the medulla of a mule dead of tetanus, with the same results as in the preceding series.

Fifth Series. - Seven rabbits were inoculated under the skin and into the muscular tissue of the back, from the medulla of the horsa above mentioned. One died within eighteen hours, and another died within ten days, but neither of them showed any signs of tetanus. A rabbit inoculated sub durâ cerebri from the medulla of the latter on August 15th, was still living and well on September 4 th.

Sixth Series. - A rabbit, which had been inoculated under the skin directly from the horse on August 1st, was eight days afterwards inoculated sub durd cerebri from the medulla of the last rabbit of the third series. It became sick, and died promptly of tetanus within the shortened period; a rabbit inoculated sub dura cerebri from its medulla showed signs of tetanus within twenty hours, but did not die until five days after inoculation.

Seventh Series. - Six rsbbits were inoculated sub durâ cerebri from emulsions of spinal cords of rabbits, which had died of tetanus within the shortened period above mentioned. These cords had been treated in a manner similar to that employed by Pasteur for the attenuation of the virus of hydrophobia, during periods varying from three to fifteen days. Five of them died of marked tetanus, the symptoms appearing and death occurring within periods longer than those of the corresponding rabbits from which the medulla had been taken, and usually proportional to the length of time the cord had been drying. One of the six showed doubtful symptoms, but nevertheless very promptly died.

Eighth Series. - A rabbit was inoculated sub durâ cerebri from the medulla of a rabbit which had died after inoculation from the cord, which hal been fifteen days drying. It showed the first signs of tetanus in forty hours, and it died of tetanus seven days after inoculation. A rabbit was inoculated sub durû cerebri from the cord which had been drying fourteen days, and it died of tetanus in twenty hours. A rabbit and a cow were inoculated sub durâ cerebri from its medulla. The former quickly died of marked tetanus. The latter died without marked symptoms within two days, and from the necropsy it seemed probable that injury to the brain had been the cause of death (there had been great difficulty in performing the operation of inoculation). A young rabbit inoculated sub durit cerebri from this cow's medulls died within sixteen hours, but showed no sigus of tetanus, and another rabbit inoculated sub dura cerebri from the medulla of this rabbit on August 27th, was still living and quite well on September 4th, never having shown any signs of illness.

Ninth Series. - Three rabbits were inoculated sul durâ cerebri on September 1st, from spinal cords of tetanus wlich had been drying respectively twenty-three, twonty-seven, and twonty-eight days (those cords were the same as those which had been drying longest, men. tioned in the preceding sevonth series). The rabbits inoculated from the twenty-three and twenty-eight day cords showed no signs of illness up to the time of the last observation, September 4 th. The one inoculated with the twenty-seven day cord for the first time showed stiff jaws and difficulty in eating on the afternoon of September 4 th.

Tenth' Series. - Threo rabbits which had been inoculated under the skin on August 18th, and had remained perfectly well, were inoculated $s u b$ durâ cerebri on September 1st, from the same cords mentioned in the ninth series. The rabbit inoculated with the twenty-three day cord was found dead the next day, but it showed no signs of tetanus either externally or at the necropsy. That of the twenty-eight day cord showed stiff jaws and would not eat for the first time on the afternoon of September 4th. That of the twenty-seven day cord showed no sign of illness up to the last observation, September 4 th.

Eleventh Series. - Three rabbits were inoculated sub durâ cerebri on August 31st, from cultures started from the horse's brain August 15th, and renewed once, namely, on August 20th. One of them had remained quite well up to the last observation, September 4th. One remained quite well until the afternoon of September $2 \mathrm{nd}$, when it showed intermittent trismus and indisposition to eat. This condition continued up to the date of the last observation, September 4th. One showed for the first time slight signs of tetanus on September 3rd, and had them also up to the date of the last observation, September 4 th.

Twelfth Series. -A trial attempt was kindly made for me by Dr. L. Wolf, Demonstrator of Medical Chemistry in the Jefferson Medical College, to isolate a ptomaine from the brain medulla and cord of the mule and cow above mentioned. The Stass-Otto method was more or less closely followed. The product obtained from the mule was injected under the skin of the back of two rabbits. They became very ill within twenty minutes, being slightly paralysed and exceedingly restless, frequently getting down flat on the belly, and up again and jerking the hind legs up; but they strowed no marked convulsive morement or trismus. They entirely recovered within six hours. The product obtained from the cow produced but little and only very transient and indefinite effects.

Note. - Several necropsies of the tetanus animais were made, and they invariably showed intense congestion of the lungs, trachea, and kidneys. Sometimes there was congestion, oftentimes none at all, of the central cerebro-spinal nervous system. The mucous membrane of the stomach was apparently normal.

Conclusions drawn from the Author's Personal Rescarches: 1. Traumatic tetanus of the horse and mule io, at least sometimes, if not always, an infectious disease, transmissible to other animals, and therefore possibly also to man ; and during the progress of this dis. ease a virus is elaborated and multiplied which is capable of producing the same infectious disease in some other animals when placed beneath the dura mater of the cerebrum.

2. This virus is contained in the medulla and spinal marrow of the animal suffering the disease. It is, like the virus of hydrophobia, capable of being strengthened in virulency by inoculation sub dur $\hat{a}$ cerebri from rabbit to rabbit, and, like the virus of hydrophobia, is capable of attenuation by exposure for a sufficient time to the action of dry air at a temperate or summer heat; and, still again like the rabic virus, its effects are far more intense when the virus is inserted beneath the dura mater of the brain than when injected beneath the skin or between the muscles of the back.

3. The author reserves bis conclusion concerning a prophylactic effect of inoculation of the attenuated virus until the completion of experiments which are at present in progress.

Conclusions drawn from the Author's Experiments when correlated with those of Nicolaicr, Carle and Antone, Rosenbach, Ferrari, Flïgge, Hochsinger, and others. - Traumatic tetanus of the lower animals and of men, at least sometimes, possibly always, is a specific infectious disease due to the action of a specific infectious virus which exists in the tissues at the seat of infection, in the blood and in the central cerebro-spinal nervous system.

In view of the experimental evidence which we possess at present, and of many unassailable observations of many surgeons and veterinarians, there seems to be ample warrant for the admission that not infrequently tetanus in man is acquired directly and indirectly from some of the domestic animals, notably the horse, which surround him.

Ambulance Classes. - Dr. W. J. Smith, of Rotherham, has been presented by the members of the ambulance class connected with the Low Stubbin Colliery, Rawmarsh, with a silver claret.jug. Mr. New. bould, by whom the presentation was made, in speaking of the value of ambulance training to colliers, referred to a case which had recently occurred, where an extemporised tourniquet was applied to stay pro. fuse bleeding following injury to the foot of a patient, who was sub. sequently taken a distance of four miles in the ambulance carriage to Rotherham Infirmary, where amputation was performed, the man making a rapid recovery. Dr. Smith, in expressing his thanks, stated that an ambulance stretcher, chair, and carriage complete, invented by Lady Alice Fitzwilliam, had been supplied to all the collieries belonging to Earl Fitzwilliam. 